\title{
Image as a Signal: Review of the Concept of Image Frequency Estimate
}

\author{
Abdul Rasak Zubair \\ Department of Electrical and Electronic Engineering \\ University of Ibadan \\ Ibadan, Nigeria. \\ E-mails: ar.zubair@ui.edu.ng, ar.zubair@yahoo.co.uk \\ Phone: +2348023278605
}

\begin{abstract}
Images are used in medicine, communication, education, entertainment and other industries. An image is a signal. Image processing improves pictorial information for man and machine. Quality of images is measured in term of parameters such as contrast, brightness, histogram and Peak Signal to Noise Ratio. Frequency Estimate as an image parameter came into existence in 2009. A review of the concept of Frequency Estimate is presented. The results of basic Image Processing Procedures on an image are found to be functions of the Frequency Estimate of the image. The lower the Frequency Estimate of an image, the higher the Peak Signal to Noise Ratio and Gain recorded in Watermark Extraction, Image Filtering and Image Signal Sampling and Compression. During basic Image Processing Procedures, Frequency Estimate also undergoes some changes. Frequency Estimate of an image increases when the image is corrupted by noise. Image Compression, Image Enhancement and Image Restoration increase the Frequency Estimate of an image as they add "desired" or "useful" noise to the image. Frequency Estimate decreases when an image undergoes convolution such as in Image Filtering and Image Degradation. Frequency Estimate is recommended as a vital image parameter.
\end{abstract}

Keywords: Image Signal, Image Parameters, Frequency Estimate, Noise, Image Processing Procedures.

CISDI Journal Reference Format

Abdul Rasak Zubair (2019): Image as a Signal: Review of the Concept of Image Frequency Estimate.

Computing,InformationSystems,DevelopmentInformatics\&AlliedResearchJournal.Vol10No1.Pp 73-86.

Availableonlineatwww.cisdijournal.org

DOI Affix - https://doi.org/10.22624/AIMS/CISDI/V10N1P6

\section{INTRODUCTION}

Images are a vital and integral part of everyday life. On an individual or person-to-person basis, images are used to warn, inform, reason, interpret, illustrate, diagnose, represent, memorize, educate, communicate, evaluate, navigate, survey and entertain [1]. One picture is worth more than ten thousand words with regard to information content [1]. A visual image is rich in information from the outer world; receiving and analysing such images is part of the routine activity of human beings throughout their lives. It is often said that the eye is the king of the human body. In other words, sight is a human being's principal sense [1]. Gray-Level Resolution is the number of gray levels in the image. The more the number of gray levels, the greater the gray-level resolution and the better the image in appearance; the more the number of bits required to represent the brightness of a pixel and the greater the cost of storage and processing. In a binary image, each pixel assumes one of two discrete values 0 or 1 that represent black and white respectively. In a gray level image, each pixel assumes one of 256 discrete values. In some systems, 0 stands for black, 255 stands for white and other digits between 0 and 255 stands for shades of gray. 
These digits are often normalized such that 0 stands for black, 1 stands for white and numbers in the range of 0 to 1 stand for shades of gray. A gray level image with $\mathrm{MN}$ pixels is stored as an $\mathrm{M}-\mathrm{by}-\mathrm{N}$ data array in a digital computer. In the RGB color model, any color can be defined using the weighted sum of additive primary colors: Red, Green, and Blue. Therefore, a color image with MN pixels is therefore stored as an M-by-N-by-3 data array in a digital computer. In the early days of computing, data was numerical. Today, data has many other forms such as voice, music, speech, images, and video. Each of these types of data is a signal. Generally, a signal is a detectable sign or notice given especially for the purpose of conveying warning, direction or information [2].

Image Processing improves pictorial information for human perception and process image data for storage, transmission, and representation for autonomous machine perception. Digital Image Processing includes operations involving digital images such as acquisition, storage, retrieval, translation, and compression. Quality of Images is measured with the aid of certain image parameters such as contrast, brightness, histogram and Peak Signal to Noise Ratio. An additional image parameter known as Image Frequency Estimate was introduced in 2009 in [3]. It has been utilized in some publications $[4,5]$.

\subsection{The Research Question}

A student sent a research question via email with regard to the Frequency Estimate of an image as follows: "Dear Authors, My name is Linh. I'm from Vietnam. I am currently a final year student at the Military Technical Academy of Vietnam. And I'm finishing my college graduation project. The topic is "Research on methods of filtering applications in medical imaging and writing simulation programs using Matlab". I consulted the document you wrote: "Development of Statistics and Convolution as Tools for Image Noise Suppression: Statistical Performance Analysis of Spatial Filters " on the internet. And there is some problem I do not understand in this document. These are: What is the Frequency Estimate associated with the image quality after filtering? And how do we calculate this parameter in Matlab (code)? I look forward to your feedback. Thank you so much. Best regard." Linh, April 2018.

\subsection{Review of the concept of Frequency Estimate}

In this work, the concept of Frequency Estimate of an image is reviewed and recommended for adoption to the image processing research community. What effects does Frequency Estimate has on basic image processing procedures? What effects do basic image processing procedures have on Frequency Estimate of an image? The significance of the concept of Frequency Estimate is $\mathrm{x}$-rayed for the benefit of the questioner and other researchers.

\section{REVIEW OF EXISTING IMAGE PARAMETERS}

Quality of an image is expressed quantitatively in the form of certain image parameters. These parameters include Contrast, Brightness, and Histogram. After Image enhancement $[1,6,7,8,9,10]$, changes in the values of these parameters reveal the change in image quality. These parameters are defined in this section and are illustrated in Figs. (1) and (2).

\subsection{Contrast}

The Contrast of an image is the range from the darkest regions of the image to the lightest regions and is given by Eqn. (1) $[1,6,7,8,9,10]$. High-contrast images have large regions of dark and light. Images with good contrast have a good representation of all luminance intensities. As the contrast of an image increases, the viewer perceives an increase in detail. This is purely a perception issue as the amount of information in the image does not increase. Human beings' perception is sensitive to luminance contrast rather than absolute luminance intensities $[1,6,7,10]$. For a color image, the Contrast is evaluated for each color component; the image Contrast is the mean of the Contrast values for the three color components. 
Contrast $=\frac{I_{\max }-I_{\min }}{I_{\max }+I_{\min }}$

where $I_{\min }$ and $I_{\max }$ are the minimum and maximum intensities respectively of a region or image.

\subsection{Brightness}

The Brightness of an image $I(m, n)$ is defined as the average of all the pixels within the image as given by Eqn. (2). $M$ and $\mathrm{N}$ are the numbers of rows and columns respectively and $\mathrm{MN}$ is the total number of pixels in $\mathrm{I}(\mathrm{m}, \mathrm{n})[1,6,7,10]$. For a color image, Brightness is evaluated for each color component; image Brightness is the mean of the Brightness values for the three color components.

$$
\text { Brightness }=\frac{1}{M N} \sum_{m=1}^{M} \sum_{n=1}^{N} I(m, n)
$$

\subsection{Histogram}

Histogram $\mathrm{h}$ of a digital image with gray levels in the range $[0, \mathrm{~L}-1]$ is a discrete function given by Eqn. (3). Normalized histogram $h_{n}$ is given by Eqn. (4) $[1,6,7,10]$. All components of a normalized histogram add up to 1 . Histogram is the number of times that a pixel with a particular gray-level occurs within an image. It provides us with a useful statistical representation of the image. An image whose histogram shows a uniform distribution over the range of gray levels is the best since this enables the eye to comprehend the contribution made by all the gray levels to the image. In practice, however, the resulting histogram is often non-uniform, with a few gray levels dominating over others to the extent that full image interpretation by the eye cannot be achieved. For a color image, $h$ is evaluated for each color component; $h$ for the three color components are plotted on the same graph as illustrated in Fig 2 (c) and (d).

$h\left(r_{k}\right)=n_{k} \mathrm{k}=0,1,2, \ldots, \mathrm{L}-1$

where $L$ is $256, r_{k}$ is the $k^{\text {th }}$ gray level and $n_{k}$ is the number of pixels in the image having gray level $r_{k}$.

$$
h_{n}\left(r_{k}\right)=\frac{n_{k}}{M N}=\mathrm{p}\left(\mathrm{r}_{\mathrm{k}}\right) \mathrm{k}=0,1,2, \ldots, \mathrm{L}-1
$$

where $\mathrm{MN}$ is the total number of pixels in the image and $p\left(r_{k}\right)$ gives an estimate of the probability of occurrence of the gray level $r_{k}$. 


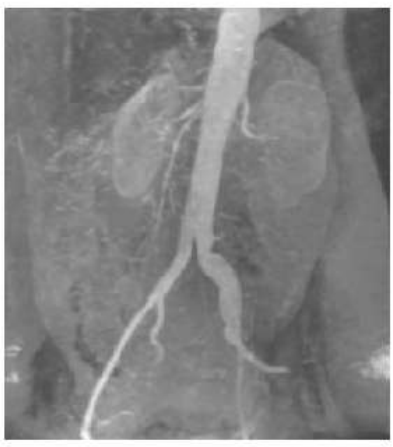

Contrast $=0.58$

Brightness $=125.70$

(a) Roots (Original)

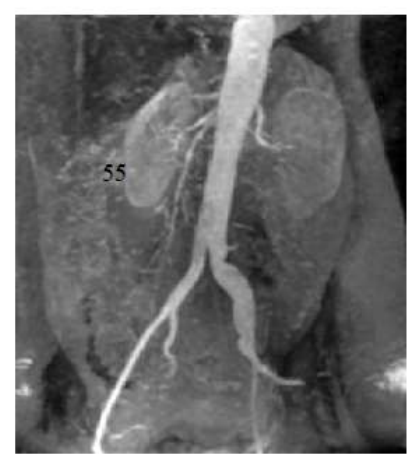

Contrast $=1$

Brightness $=127.78$

(b) Roots (Enhanced by Auto-scaling)

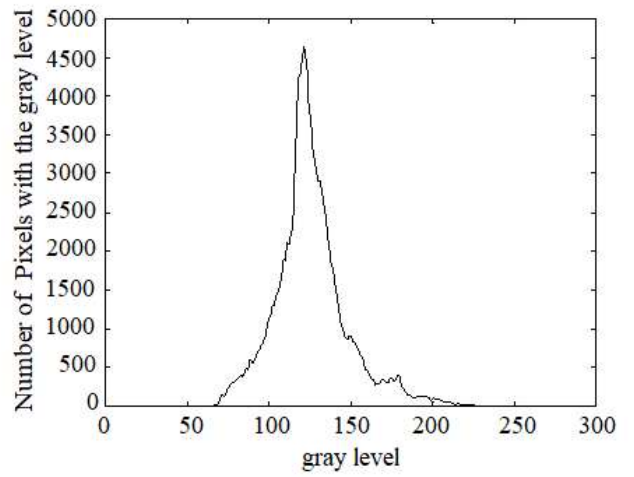

(c) Histogram of Roots (Original)

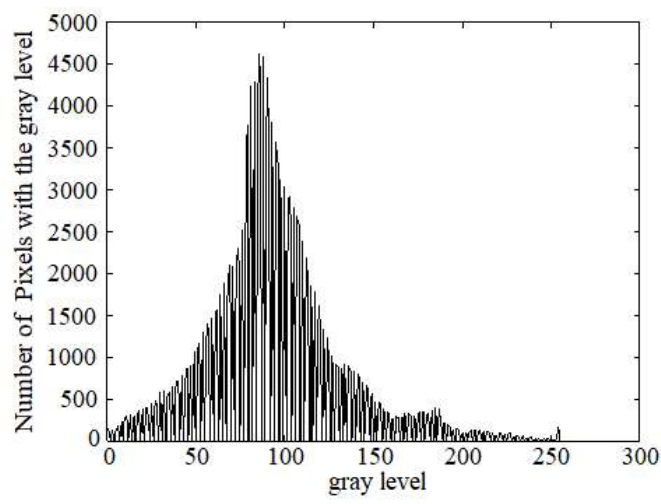

(d) Histogram of Roots (Enhanced by Auto-scaling)

Figure 1: Contrast, Brightness and Histogram for Gray Level Images [10]

\subsection{Performance Metric}

The Peak Signal to Noise Ratio (PSNR) is a measure of the degree of corruption or degradation of an image with noise or blurring [3,4,5,11]. Eqn. (5) defines $\mathrm{PSNR}_{\mathrm{c}}$ which compares the corrupted or degraded image $\mathrm{g}$ with the true image $f$. The lower the PSNR ${ }_{c}$, the higher the degree of corruption or degradation. Eqn. (6) gives PSNR $r$ which compares the filtered or recovered image $f_{r}$ with the true image $f$. The Gain of the filter is given by Eqn. (7). The higher the PSNR $r$ and Gain, the higher the noise suppression by the filter.

$$
P S N R_{c}=10 \log _{10}\left[\frac{255^{2}}{\frac{1}{c M N}\left[\sum_{y=1}^{M} \sum_{x=1}^{N} \sum_{z=1}^{c}(g(y, x, z)-f(y, x, z))^{2}\right]}\right]
$$

where $\mathrm{c}$ is 1 for gray level image and $\mathrm{c}$ is 3 for an RGB color image. 

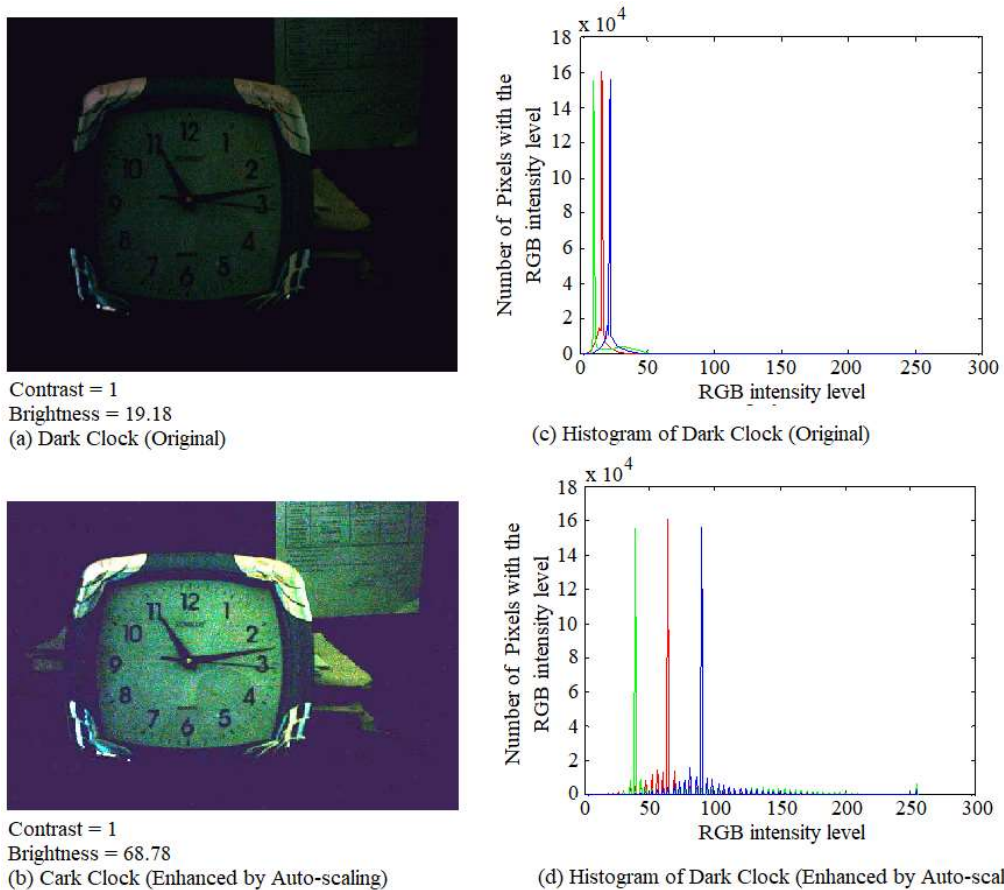

(b) Cark Clock (Enhanced by Auto-scaling)

(d) Histogram of Dark Clock (Enhanced by Auto-scaling)

Figure 2: Contrast, Brightness and Histogram for RGB Color Images [10]

$P S N R_{r}=10 \log _{10}\left[\frac{255^{2}}{\frac{1}{c M N}\left[\sum_{y=1}^{M} \sum_{x=1}^{N} \sum_{z=1}^{c}\left(f_{r}(y, x, z)-f(y, x, z)\right)^{2}\right]}\right]$

Gain $=P S N R_{r}-P S N R_{c}$
(6)

(7)

\section{THE CONCEPT OF IMAGE FREQUENCY ESTIMATE}

\subsection{Introduction of the Concept of Frequency Estimate}

In [3], During the conversion of an RGB color image to an index image and back to an RGB image which is a reversible image compression process/color sampling process, it was observed that better result was obtained for images with the lower rate of change of intensity. There was a need for an additional image parameter to account for this observation. An average measure of the rate of change of intensity in an image was required as an image parameter. This can be borrowed from the concept of frequency of a periodic signal. An analog periodic signal $v(t)$ of Eqn. (8) shown in Fig. 3 is said to have period T seconds and frequency $\mathrm{f} \mathrm{Hz}$. The magnitude of the signal rises from 0 Volt to maximum ( $V_{m}$ Volt), falls back to 0 Volt, falls further to minimum (- $V_{m}$ Volt) and then rises back to 0 Volt; this cycle is repeated. Period $T$ is the time it takes to complete a cycle while $f$ is the number of cycles in one second. $T$ and $f$ are related as in Eqn. (9). The frequency of a signal is an important parameter of the signal. 


$$
\begin{gathered}
v(t)=V_{m} \operatorname{Sin}(2 \pi f t) \\
T=\frac{1}{f}
\end{gathered}
$$

An image is a signal. In an image signal, the intensity varies from pixel to pixel along rows and columns. This variation may not be periodic as in Fig. 3. In 2009, the concept of Image Frequency Estimate was introduced in [3] as an average measure of variation of intensity along rows and columns as described by Eqns. (10), (11) and (12) [3,4,5]. The image $\mathrm{I}$ is an M-by-N-by-c matrix. $\mathrm{c}$ is 1 for gray level image and $\mathrm{c}$ is 3 for an RGB color image. $y$ is the row axis, $\mathrm{x}$ is the column axis, and $\mathrm{z}$ is the color axis.

$\begin{aligned} & \text { Frequency } \\ & \text { Estimate }\end{aligned}=\frac{1}{c M(N-1)} \frac{\partial I}{\partial x}+\frac{1}{c N(M-1)} \frac{\partial I}{\partial y}$

where

$$
\frac{\partial I}{\partial x}=\sum_{z=1}^{c} \sum_{y=1}^{M} \sum_{x=2}^{N}|I(y, x, z)-I(y, x-1, z)|
$$

and

$$
\frac{\partial I}{\partial y}=\sum_{z=1}^{c} \sum_{x=1}^{M} \sum_{y=2}^{N}|I(y, x, z)-I(y-1, x, z)|
$$

Eqn. (11) gives the sum of absolute differences between two adjacent pixels' intensities along the rows while Eqn. (12) gives the sum of absolute differences between two adjacent pixels along the columns. Therefore, Eqn. (10) is the mean of the absolute differences between two adjacent pixels along the rows plus the mean of the absolute differences between two adjacent pixels along the columns. Eqn. (10) is modified to give Eqn. (13) which gives the mean of the absolute differences between two adjacent pixels along the rows and the columns. Eqn. (13) is recommended for adoption as Frequency Estimate of an Image and is presented in a pictorial form in Fig. 4. For $\mathrm{M}=\mathrm{N}$, the result of Eqn. (10) is twice that of Eqn. (13).

$\begin{aligned} & \text { Frequency } \\ & \text { Estimate }\end{aligned}=\frac{1}{c M(N-1)+c N(M-1)}\left(\frac{\partial I}{\partial x}+\frac{\partial I}{\partial y}\right)$ 


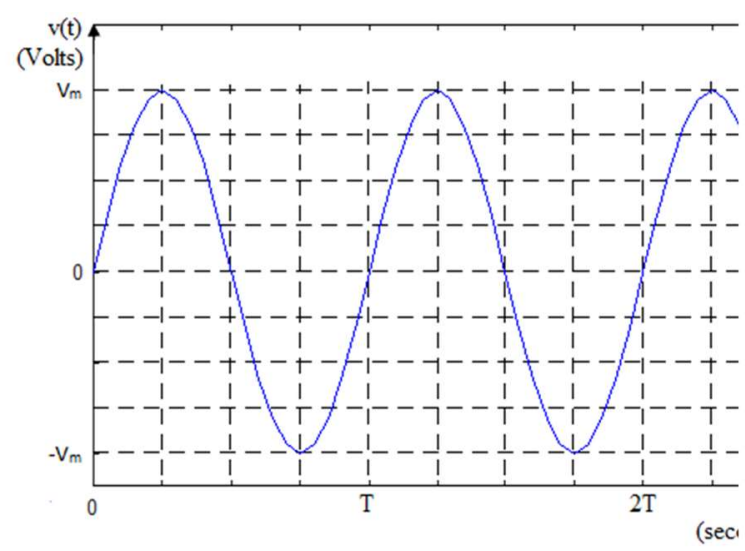

Figure 3: Analogue Periodic Signal
Frequency Estimate of an Image I is given as $\begin{aligned} & \text { Frequency } \\ & \text { Estimate }\end{aligned}=\frac{1}{c M(N-1)+c N(M-1)}\left(\frac{\partial I}{\partial x}+\frac{\partial I}{\partial y}\right)$

where

$\frac{\partial I}{\partial x}=\sum_{z=1}^{c} \sum_{y=1}^{M} \sum_{x=2}^{N}|I(y, x, z)-I(y, x-1, z)|$

$\frac{\partial I}{\partial y}=\sum_{z=1}^{c} \sum_{x=1}^{N} \sum_{y=2}^{M}|I(y, x, z)-I(y-1, x, z)|$,

I is a M-by-N-by-c matrix,

$c=1$ for gray level image and

$\mathrm{c}=3$ for rgb colour image.

Figure 4: The Expression for the Frequency

Estimate of an Image

\subsection{Computation of Frequency Estimate}

Table 1 shows an example of Image I with $\mathrm{x}$, y and $\mathrm{z}$ axis labels. $\mathrm{M}=3, \mathrm{~N}=3$, and $\mathrm{c}=3$. Table 2 illustrates the computation of Frequency Estimate for Image I of Table 1 based on Eqns. $(11,12,13)$.

\subsection{Program for the Computation of Frequency Estimate}

Fig. 5 shows the flowchart of a program to compute the Frequency Estimate of an Image I. The flow chart is coded into a Matlab program which is presented in Fig. 6 .

Table 1. A typical Image matrix I(3-by-3-by-3); $M=3, N=3$, and $c=3$

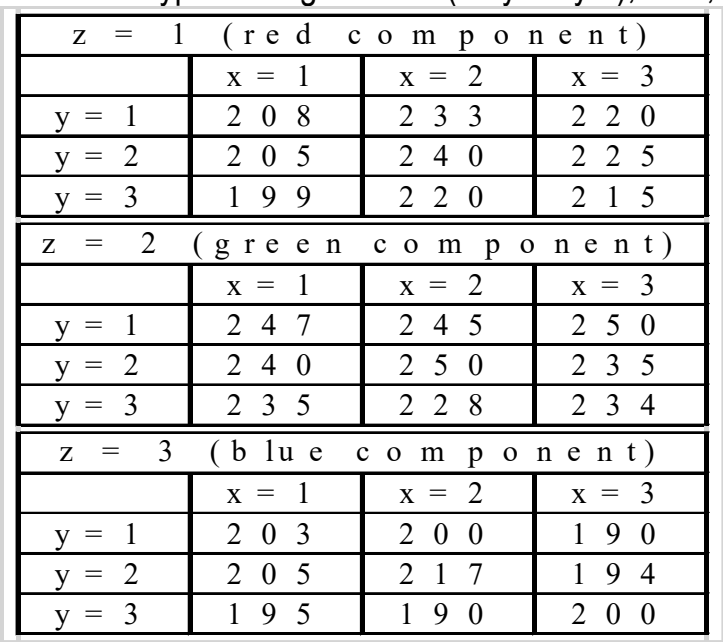




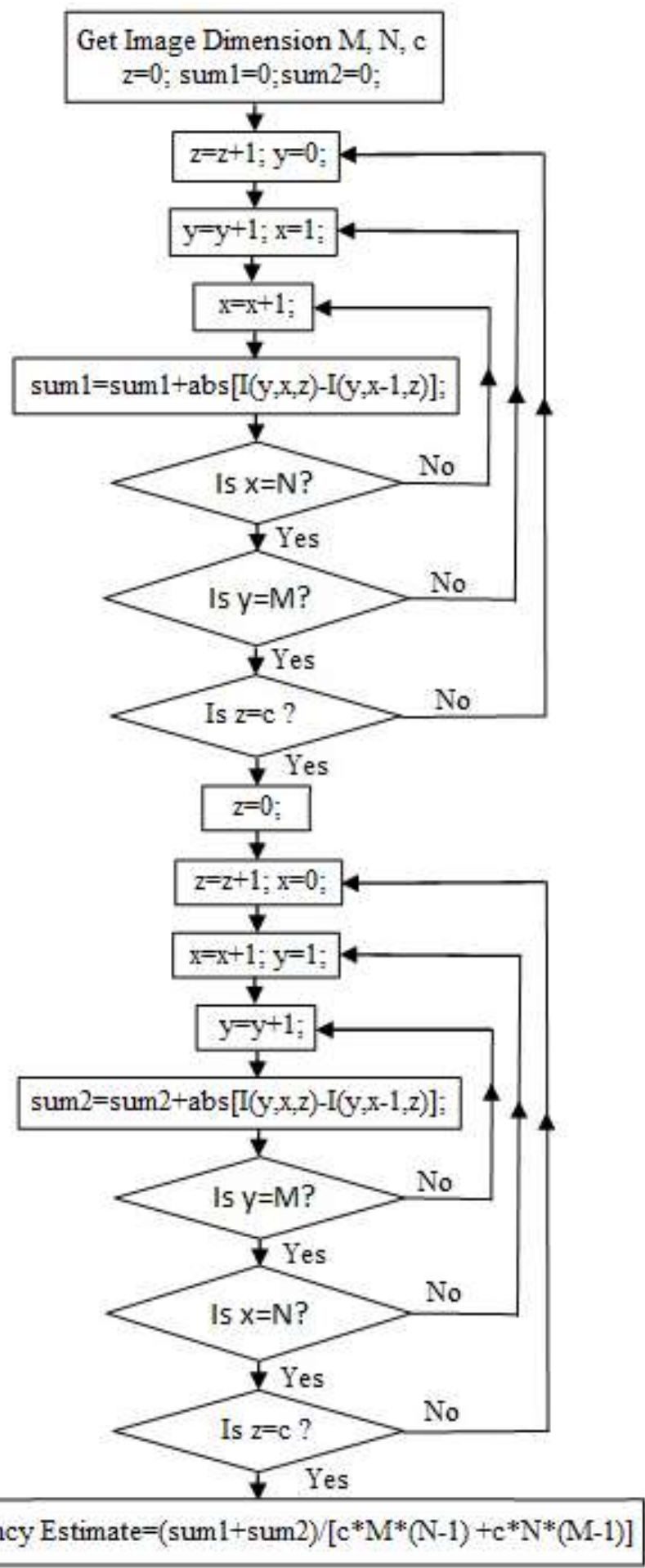

Figure 5: Flow Chart for the Computation of Frequency Estimate of Image I 
Table 2. Computation of Frequency Estimate of the matrix I of Table 1

\begin{tabular}{|c|c|c|c|c|c|c|c|c|}
\hline \multicolumn{8}{|c|}{$\begin{array}{c}\text { Absolute differences } \\
\text { betwe en two adjacent } \\
\text { pixels along the rows }\end{array}$} \\
\hline \\
\hline
\end{tabular}

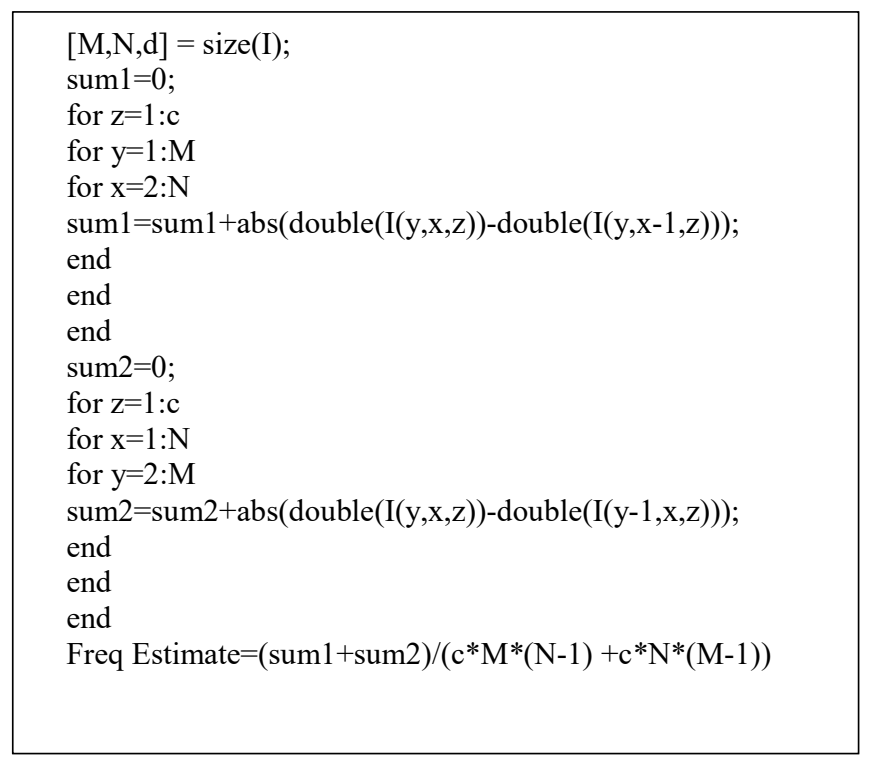

Figure 6: Matlab Program for the Computation of Frequency Estimate of Image I 


\section{THE SIGNIFICANCE OF THE CONCEPT OF IMAGE FREQUENCY ESTIMATE}

\subsection{Effect of Frequency Estimate on Image Reconstruction after Sampling or Compression}

For certain applications, it may be necessary to convert an RGB color image w (M-by-N-by-3) to index image y (M-by$\mathrm{N}$-by-1) and a color map $p$ (256 by 3 ) as in [3]. This conversion amount to both signal sampling and image compression. Given y and $p$, an RGB color image $w_{r}\left(M\right.$ by $N$ by 3 ) is recovered or reconstructed. The closeness of $w_{r}$ to $w$ is measured in term of Peak Signal to Noise Ratio (PSNR). It was found in [3] that the lower the Frequency Estimate of $\mathrm{w}$, the higher the PSNR and hence the more accurate the image reconstruction as shown in Fig. 7.

\subsection{Effect of Frequency Estimate on Robustness of Digital Watermarking}

Digital Watermarking is the hiding of one image known as watermark $w$ in another image known as host $h$ for copyright protection, content authentication, and secret communication [3]. The watermarked image $h_{\text {mkd }}$ is a combination of $h$ and $w$. During further processing, transmission or storage, $h_{m k d}$ may be attacked or corrupted. Robustness requires that watermark $w$ is detectable or recoverable from corrupted $h_{m k d}$. The recovered watermark $w_{r}$ is compared with the original watermark $w$ in term of Peak Signal to Noise Ratio (PSNR). It was found in [3] that the lower the Frequency Estimate, the higher the PSNR and hence the higher the robustness of the watermarking as illustrated in Fig. 8.

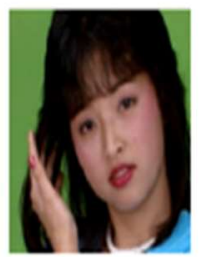

Maruda

Freq Estimate

$=3.16$

(a) Original Images

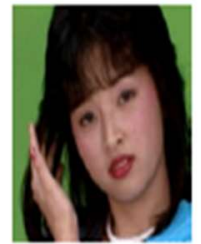

Maruda

$\mathrm{PSNR}=39.55 \mathrm{~dB}$

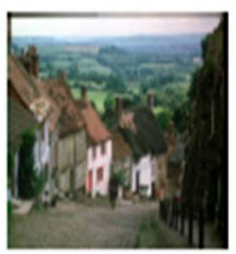

Goldhill

Freq Estimate

$=10.53$

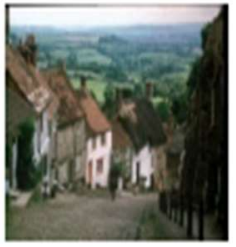

Goldhill

PSNR $=35.24 \mathrm{~dB}$

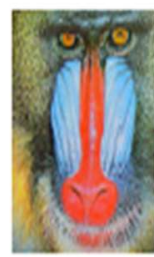

Baboon

Freq Estimate

$=24.45$

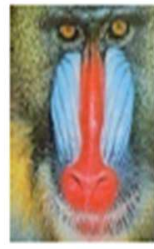

Baboon

$\mathrm{PSNR}=31.71$ (b) Corresponding Restored rgb Colour Images after Conversion from rgb to Index and back to rgb

Figure 7: Effect of Frequency Estimate on Image Reconstruction after Sampling or Compression (Fig. 4 and Table 1 in (4)]

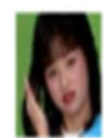

Mazuda

Freq Estimate

$=13.04$

(a) Three watermarks $(57 \times 57 \times 3)$ with different Freqency Estimate
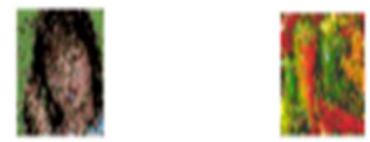

Mazuda

Peppers

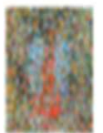

Baboon

$\operatorname{PSNR}=14.43 \mathrm{~dB} \quad$ PSNR $=13.53 \mathrm{~dB} \quad$ PSNR $=12.26 \mathrm{~dB}$

(b) Corresponding detected watermarks with q90 JPEG compression attack

Figure 8: Robustness of Watermarking against Watermark Image Frequency Estimate (Figure 15 in [4]) 


\subsection{Effect of Frequency Estimate on Spatial Filtering Gain}

Spatial filters for image de-noising are low pass filters $[4,5,6,7,8,9,10]$. In [4], thirty-nine spatial filtering algorithms were studied. The algorithms were tested for the suppression of six types of noise in a test bed of thirty-six test images. The effect of image Frequency Estimate on spatial filtering Gain was investigated in [4]. A strong correlation which is close to inverse proportionality is found to exist between the Gain and Frequency Estimate of the original image as illustrated in Fig. 9 [4]. The lower the Frequency Estimate of an original image, the higher the Gain obtainable when a corrupted version of the image is filtered. The same trend is found in Tables 2 and 5 of [5].

\subsection{Effect of Image Noise and Image De-noising on Frequency Estimate}

In this work, Images are corrupted with known quantities of Salt and Pepper Noise (SPN) [5] and Random Valued Impulse Noise (RVIN) [5]. The Frequency Estimate of the original images, corrupted images, and median filtered images are recorded and presented in Fig. 10. Image corruption with noise increases the Frequency Estimate of an image while de-noising reduces it. For example, Fig. 10(a) gives the Frequency Estimate of the original Lena image, Lena image corrupted with 5\% SPN and corresponding median filtered image as $6.40,10.37$ and 3.38 respectively. Convolution, smoothening, filtering and de-noising reduce Frequency Estimate. The same trend is observed in Fig. 10(b) to (f).

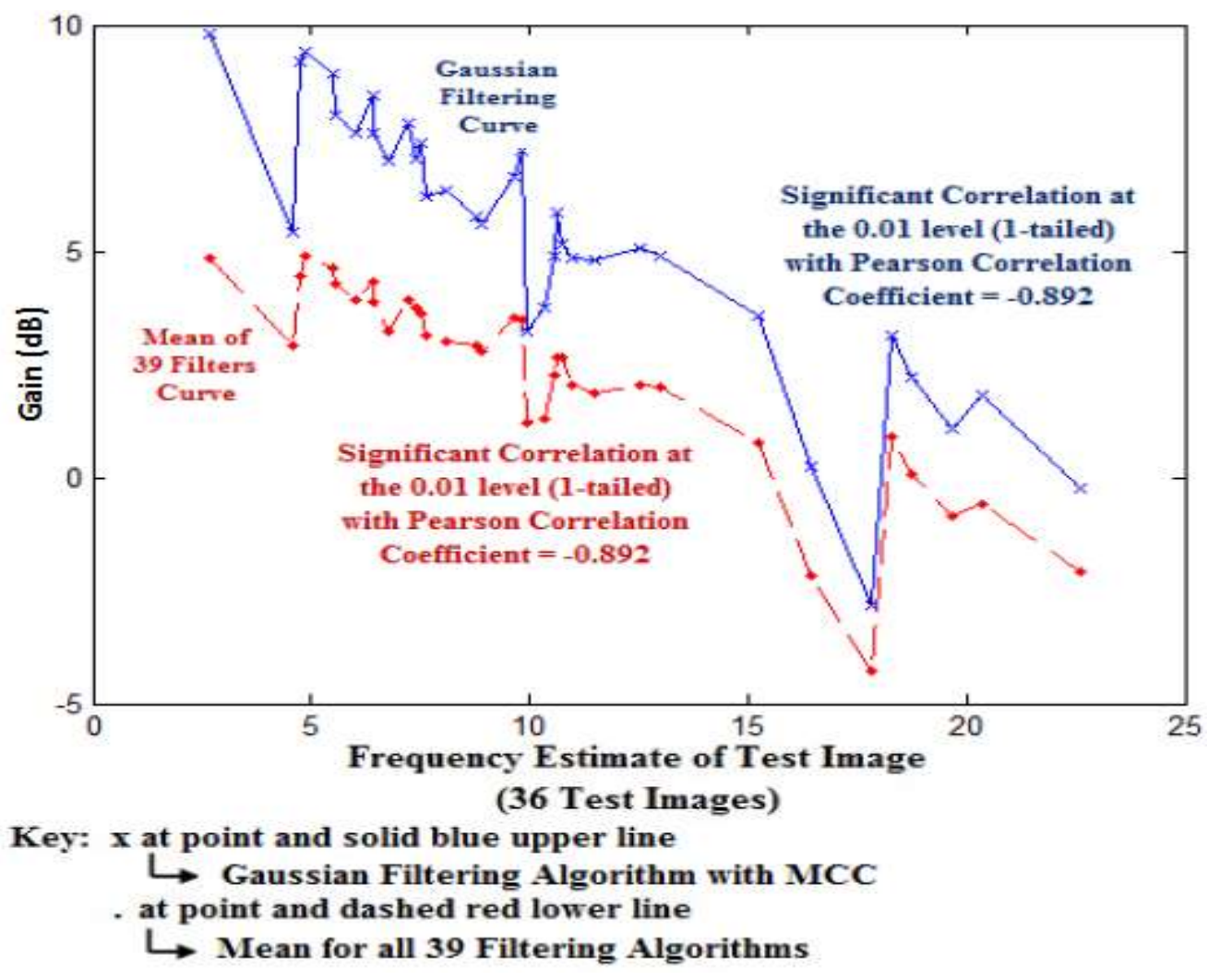

Figure 9: Spatial Filtering Gain versus Frequency Estimate (Fig. 8(b) in [4]) 

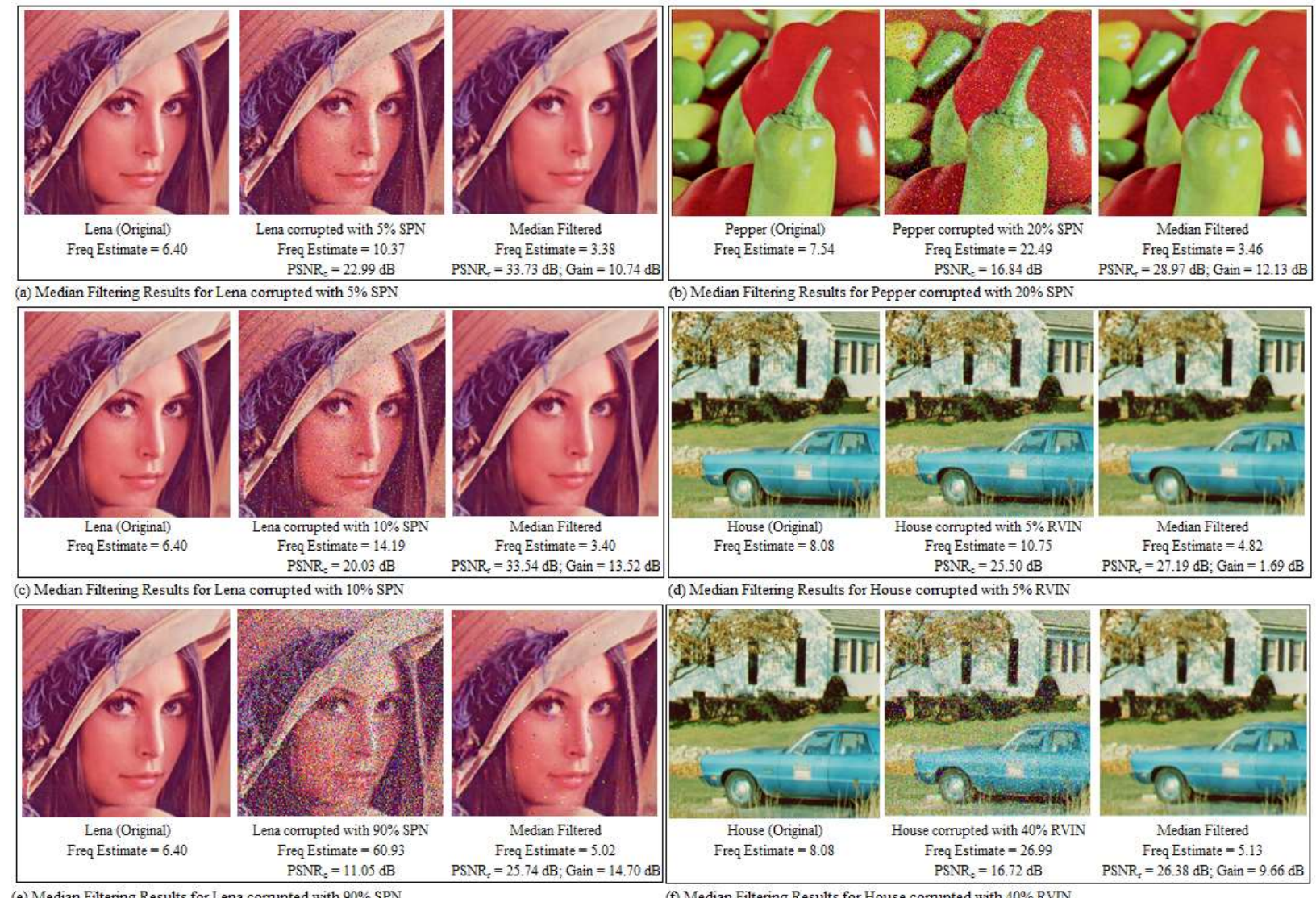

Figure 10: Effect of Noise and De-noising on Frequency Estimate

\subsection{Effect of Image Enhancement on Frequency Estimate}

Image enhancement processes an image so that the resulting image will be more suitable than the original image for a specific application $[1,6,7,8,9,10]$. Image enhancement procedure operates on an input image $f(m, n)$ to give an output image $\mathrm{g}(\mathrm{m}, \mathrm{n})$. Examples of image enhancement techniques are histogram processing, auto-scaling and point transformations such as linear, exponential, logarithm and power-law transformations [10]. Image Enhancement improves both the contrast and or brightness of an image [10]. The goal of image enhancement is beauty [12]. The effect of image enhancement on image Frequency Estimate is investigated in this work. The results are presented in Fig. 11. Image enhancement increases Frequency Estimate of an image as illustrated in Fig. 11. Image enhancement can be viewed as a corruption of an image with a "desired" or "useful" kind of noise. This accounts for the increase in Frequency Estimate of the image.

\subsection{Effect of Image Degradation and Image Restoration on Frequency Estimate}

In this work, images are blurred with known motion blur length and angle [13]. Image restoration is applied to the blurred images $[12,13]$. The Frequency Estimate of original images, blurred images, and restored images are recorded and presented in Fig. 12. Image blurring or degradation involves convolution with Point Spread Function (PSF) and it reduces the Frequency Estimate of the image as shown in Fig. 12. Fig. 12 also show that Image restoration increases the Frequency Estimate. 


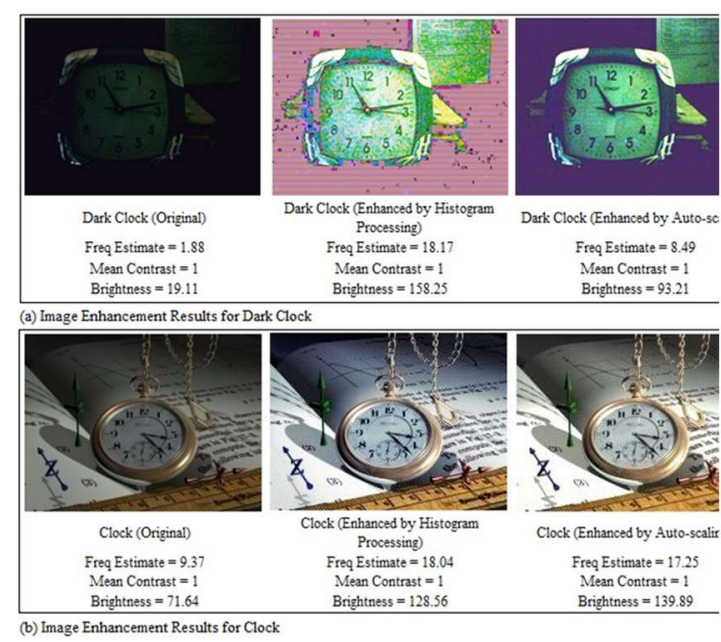

Figure 11: Effect of Image Enhancement on Frequency Estimate

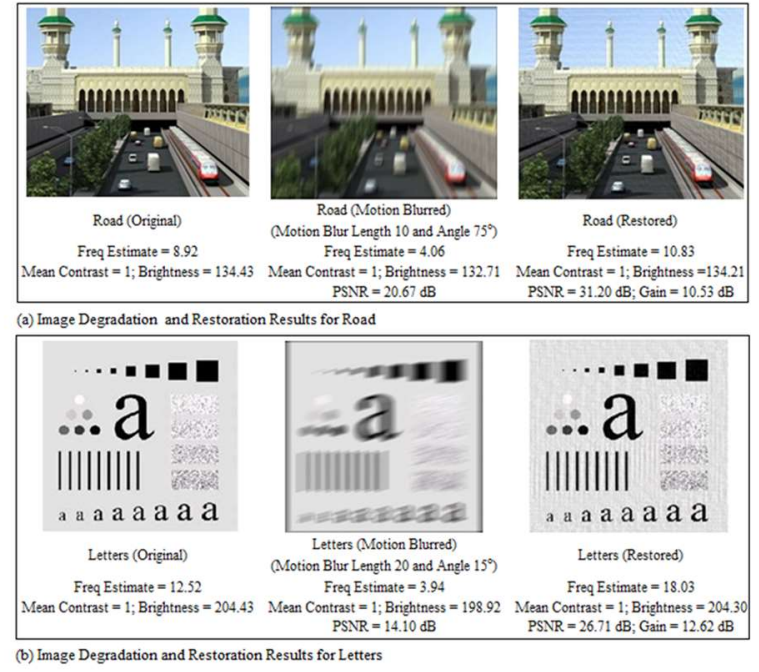

Figure 12: Effect of Image Degradation and Image Restoration on Frequency Estimate

\section{CONCLUSION}

The concept of Frequency Estimate associated with digital image has been reviewed. Frequency Estimate has been confirmed to be a very important parameter of an image. Basic Image processing procedures affect Frequency Estimate of an image. The Frequency Estimate of an image increases whenever the image is corrupted by any form of noise. Image compression, Image enhancement, and Image Restoration can be viewed as adding "desired" or "useful" kinds of noise to an image and therefore increases the Frequency Estimate of an Image. The Frequency Estimate of an Image decreases whenever the image undergoes convolution, smoothening or filtering. Image degradation involving convolution of Image with Point Spread Function (PSF) also reduces Frequency Estimate of the image. Frequency Estimate also affects the output of some image processing procedures. The Lower the Frequency Estimate, the higher the PSNR and Gain recorded in watermark extraction, filtering and image sampling/compression. 


\section{REFERENCES}

[1] R.C. Gonzalez and R. E. Woods, Digital Image Processing. Massachusetts: Addison-Wesley, 2002.

[2] J.D. Brook and R.J. Wynne, Signal Processing Principles and Applications. London: Edward Arnold, 1988.

[3] A.R. Zubair, O.A. Fakolujo and P.K. Rajan, "Digital watermarking of still images with color digital watermarks," In the IEEE EUROCON 2009, pp. 1338-1345, May 2009.

[4] A.R. Zubair and O.A. Fakolujo, "Development of Statistics and Convolution as Tools for Image Noise Suppression: Statistical Performance Analysis of Spatial Filters," IEEE Afr. J. of Comp \& ICTs, vol. 6, no. 5, pp. 95-108, 2013.

[5] A.R. Zubair and H.O. Busari, "Robustness of Median Filter for suppression of Salt and Pepper Noise (SPN) and Random Valued Impulse Noise (RVIN)," International Journal of Image Processing (IJIP), vol. 12, no. 1, pp. 12-27, 2018.

[6] B. Chanda and D.D. Majumer, Digital Image Processing and Analysis. India: Prentice-Hall, 2000.

[7] F.C. Tony and S. Jianhong, Image Processing and Analysis: Variational, PDE, Wavelet, and Stochastic Methods. Philadelphia: SIAM, 2005.

[8] A.K. Jain, Fundamentals of digital image processing. India: Prentice-Hall, 1989.

[9] A. Bovik, Handbook of image and video processing. New York: Academic, 2000.

[10] A.R. Zubair, "Comparison of Image Enhancement Techniques," International Journal of Research in Commerce, IT \& Management, vol. 2, no. 5, pp. 44-52, 2012.

[11] S.S.O. Choy, Y. Chan and W. Siu, "An Improved Quantitative Measure of Image Restoration Quality," Proc. IEEE International Conference on Acoustics, Speech and Signal Processing (ICASSP'96), vol. III, pp. 16131616, 1996.

[12] A.R. Zubair, "Spatial Domain Restoration Scheme," In the 4th International Conference on Research and Development, pp. 46-54, 2011.

[13] A.R. Zubair, "Frequency Domain Image Restoration: Parameter K (NSR) and Gain of Wiener Filter vis-à-vis Length and Direction of Motion Blur," International Journal of Computer and Information Technology (IJCIT), vol. 7, no. 2, pp. 64-73, 2018. 